\title{
Automatic Tuning and Locking of Hitless Add-Drop Filters
}

\author{
Maziyar Milanizadeh, Douglas Aguiar, Matteo Petrini, Emanuele Guglielmi, Francesco Zanetto, \\ Fabio Toso, Francesco Morichetti and Andrea Melloni \\ Dipartimento di Elettronica, Informazione e Bioingegneria - Politecnico di Milano, \\ Milano, 20133 Italy \\ e-mail: andrea.melloni@polimi.it
}

\begin{abstract}
Automatic tuning and locking of silicon microring resonator filters with hitless functionality in multichannel WDM system is presented. The filter spectral shape is optimized to match the signal spectral shape. A technique to cancel thermal crosstalk effects is exploited to automatically create filters lookup table.
\end{abstract}

Keywords: Automatic tuning algorithm, lookup table, thermal crosstalk, add-drop multiplexer, microring filters

\section{Introduction}

The automatic tuning and control of Photonic Integrated Circuits (PICs) to implement flexible, hitless and reconfigurable devices for telecom and interconnect applications during their operation in a multichannel WDM system is one of the hot topics in integrated photonics. Here we consider an advanced ring-based filter with hitless operation, investigate the tuning techniques, present a technique to cancel the thermal crosstalk between the various elements of the PIC and show how to create a filter lookup table adapted to the modulated signal automatically.

\section{Tuning and locking of hitless filter}

The schematic of the considered silicon photonics filter is shown in Fig. 1a and a photo in Fig. 1b. The silicon channel waveguide is $490 \mathrm{~nm}$ wide and is buried in a silica cladding. The filter has $1 \mathrm{THz}(8 \mathrm{~nm}) \mathrm{FSR}, 40 \mathrm{GHz}$ $3 \mathrm{~dB}$ bandwidth, and $20 \mathrm{~dB}$ in-band isolation. Hitless operation is achieved by switching the $\mathrm{MZ}$ tuneable coupler connecting the filter to the line bus. This allows reaching a high degree of isolation during the filter tuning.

A MZ-based channel labeller is integrated on chip at the Add port allowing to distinguish the operating channel at the monitor in presence of other WDM channels. This labeler is typically operated at few $\mathrm{kHz}$ and provides an intensity modulation of few percent $(<8 \%)$ to the channel inserted in the Add port which can be extracted from the readout signal of the monitor. This label does not impair the system quality [4]. Sub-band gap transparent detectors (CLIPP) [1] are placed at the Drop port providing the feedback signal used by the control algorithms which are embedded in a FPGA that drives the five integrated heaters [4].

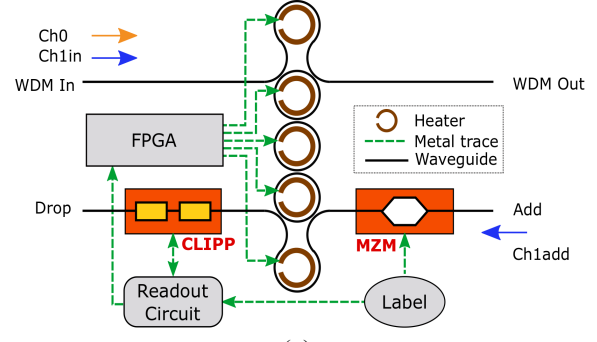

(a)

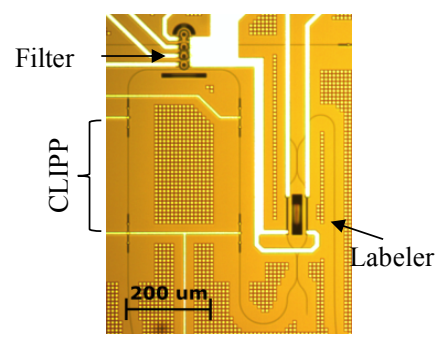

(b)

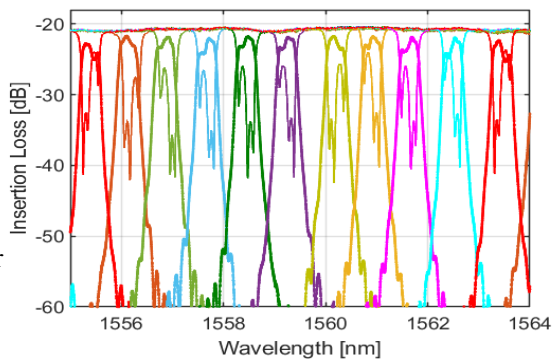

(c)

Figure 1: (a) Functional block diagram of the reconfigurable hitless filter along the relevant control elements adopted to demonstrate fine tuning and automatic look up table creation b) Filtering unit including monitoring CLIPP and on-chip labeler to mark the add signal (c) Through and Drop port detailed transfer function of filters for automatically created lookup table on 10 channels $100 \mathrm{GHz}$ apart.

\section{Thermal cross-talk free tuning technique}

Recently we proposed a novel technique, named Thermal Eigenmode Decomposition (TED) [3], which can be adopted in generic control algorithms to cancel out the effects of thermal crosstalk for arbitrary PICs.

TED technique requires all the actuators to be controlled simultaneously according to appropriate weights, which are chosen based on eigensolution of the thermally coupled system. Fig. 2(a) shows a top view microphotograph of a 3-ring based filter fabricated in silicon oxynitride ( $\mathrm{SiON}$ ) technology with individual heaters (details in [3]). The output optical power is measured via an external photodiode and transmitted to a PC where a control algorithm defines the control signals to be applied to the actuators. Phase perturbations were intentionally introduced in every MRR of the SiON filter by applying random errors in the voltages driving the heaters around their optimum tuning point as large as $100 \mathrm{pm}(12.5 \mathrm{GHz}$ versus $6.5 \mathrm{GHz} \mathrm{BW}$ of the filter). A $5 \mathrm{Gbit} / \mathrm{s}$ OOK modulated signal (with 
carrier wavelength of $1565.470 \mathrm{~nm}$ ) was used and the TED based tuning algorithm was targeted to minimize the output power at the Through port. Transfer function of converged filter for Through and Drop port is presented in Fig. 2(b). To illustrate the improvement in tuning performance, TED based method was then compared to the individual tuning in terms of convergence ratio and speed. Fig. 2(d), shows that in many cases sequential tuning of individual resonators did not converge to the target filter shape and a poor Through port isolation with deep oscillations was obtained. In contrast, the TED-based tuning did converge for all the considered initial cases in less than 40 iterations (see Fig. 2(c)).

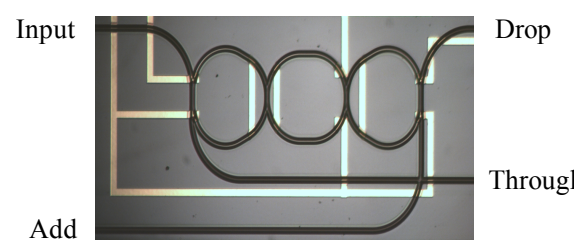

(a)
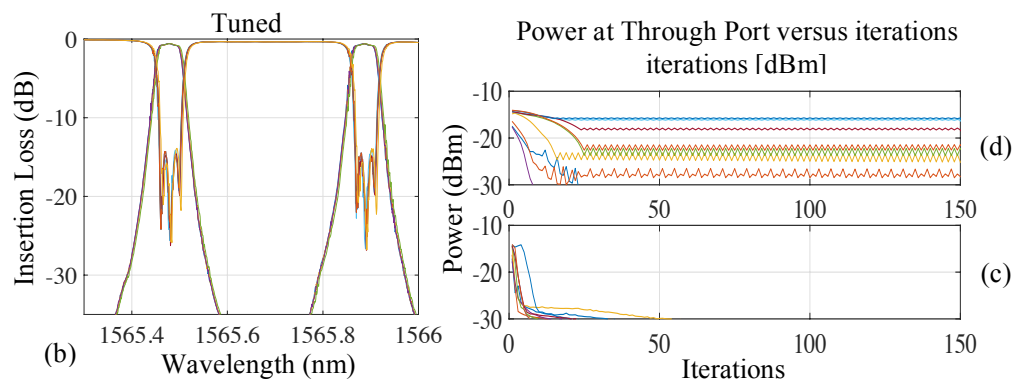

Figure 2: Experimental validation of automatic tuning based on TED method. (a) Top view photograph of a 3rd order coupled MRR filter fabricated in SiON technology. (b) Measured transmission of the Through and Drop port of the filter. (c) Convergence rate of TED based tuning for 10 random cases versus (d) individually tuning of the MRRs starting from the same random perturbations.

\section{Automatic lookup table generation}

A tuning algorithm based on the TED technique is adopted to control the tuneable optical signal source to automatically create a lookup table for the add/drop filter. The optical modulated signal (100 Gbit/s QPSK) is tuned at the relevant wavelength and an electronic board executes the tuning algorithm to find the parameters of the filter for that channel. This is repeated for every channel of the ITU grid and the look up table is created based on the performance requirements of the filter. A microscopic picture of the fabricated chip including the labelling $\mathrm{MZ}$ is presented in Fig. 1(b). In [4] we demonstrate tuning and locking of such filter in presence of DWDM channels on the grid using TED based algorithm.

Adopting this technique, ten different channels spaced by $100 \mathrm{GHz}$ have been tuned in $1 \mathrm{THz}$ range and the lookup table created. The transfer functions are shown in Fig. 1(c). Thermal perturbations from the neighbouring filters (along their tuning process) can be accounted while tuning the filters to automatically find their fined tuned conditions and create the relevant look up table. Upon the need, these lookup tables can adapt themselves to new conditions of the chip automatically.

This work was supported in part by the EU H2020 Program under Grant STREAMS (no. 688172)

\section{REFERENCES}

[1] F. Morichetti et al.,"Non-invasive on-chip light observation by contactless waveguide conductivity monitoring," JSTQE, $20,4,2014$.

[2] J. C. C. Mak, W. D. Sacher, T. Xue, J. C. Mikkelsen, Z. Yong and J. Poon, "Automatic Resonance Alignment of High-Order Microring Filters," Quantum Electronics, vol. 51, no. 11, pp. 1-11, 2015.

[3] M. Milanizadeh, D. Aguiar, A. Melloni, and F. Morichetti, "Cancelling thermal cross-talk effects in photonic integrated circuits," Journal of Lightwave Technology, 2019. DOI: 10.1109/JLT.2019.2892512

[4] D. Aguiar, M. Milanizadeh, E. Guglielmi, F. Zanetto, G. Ferrari, M. Sampietro, F. Morichetti, and A. Melloni, "Automatic Tuning of Silicon Photonics Microring Filter Array for Hitless Reconfigurable Add-Drop,” Journal of Lightwave Technology, 2019. 